\title{
Magnetization reversal signatures in the magnetoresistance of magnetic multilayers
}

\author{
P. Perna,,${ }^{1,}$ C. Rodrigo, ${ }^{1,2}$ M. Muñoz,${ }^{3,4}$ J. L. Prieto, ${ }^{4}$ A. Bollero, ${ }^{1}$ D. Maccariello, ${ }^{1,2}$ J. L. F. Cuñado,${ }^{2}$ M. Romera, ${ }^{4}$ \\ J. Akerman, ${ }^{4}$ E. Jiménez, ${ }^{2}$ N. Mikuszeit, ${ }^{1,2}$ V. Cros, ${ }^{5}$ J. Camarero, ${ }^{1,2, \dagger}$ and R. Miranda ${ }^{1,2}$ \\ ${ }^{1}$ Instituto Madrileño de Estudios Avanzados en Nanociencia IMDEA-Nanociencia, \\ Campus Universidad Autónoma de Madrid, 28049 Madrid, Spain \\ ${ }^{2}$ Departamento de Fisica de la Materia Condensada and Instituto "Nicolás Cabrera," \\ Universidad Autónoma de Madrid, 28049 Madrid, Spain \\ ${ }^{3}$ IMM-Instituto de Microelectrónica de Madrid (CNM-CSIC), Isaac Newton 8, PTM, E-28760 Tres Cantos, Madrid, Spain \\ ${ }^{4}$ Instituto de Sistemas Optoelectrónicos y Microtecnología (ISOM), Universidad Politécnica de Madrid, 28040 Madrid, Spain \\ ${ }^{5}$ Unité Mixte de Physique CNRS/Thales and Université Paris Sud 11, 91767 Palaiseau, France
}

(Received 20 July 2011; revised manuscript received 27 June 2012; published 16 July 2012)

\begin{abstract}
The simultaneous determination of magnetoresistance and vectorial-resolved magnetization hysteresis curves in a spin valve structure reveals distinct magnetoresistive features for different magnetic field orientations, which are directly related to the magnetization reversal processes. Measurements performed in the whole angular range demonstrate that the magnetoresistive response originates from the intrinsic anisotropic angular dependence of the magnetization orientation between the two ferromagnetic layers. This also provides direct proof that the spin-dependent scattering in the bulk of the magnetic layers is at the origin of the magnetoresistive signal.
\end{abstract}

DOI: 10.1103/PhysRevB.86.024421 PACS number(s): 75.47.-m, 75.60.Jk, 75.75.-c, 85.75.-d

Large magnetoresistance (MR) effects observed in ferromagnetic (FM) layers separated by nonmagnetic (NM) spacers have attracted sustained interest over the past decades for both fundamental and technological reasons. ${ }^{1,2}$ The effects originate from spin-dependent scattering processes affecting electrons that travel across multilayered structures with different relative magnetization orientation between adjacent FM layers. ${ }^{3}$ To observe large MR responses, one has to reorient the magnetization of the FM layers relative to one another, either by applying external magnetic fields (i.e., direct magnetic torque on the local magnetization) $)^{1,5}$ or by injecting spin polarized currents (i.e., via transfer of angular momentum between the spin polarized conduction electrons and the local magnetization). ${ }^{6,7}$ The maximum MR value is expected when the magnetic configuration of the FM layers reorients from a fully parallel (P) to a fully antiparallel (AP) configuration.

Even though it is commonly assumed that the MR depends on the magnetic anisotropy of the multilayer structure, clear experimental proof of the direct relationship between the magnetoresistive behavior and the magnetization reversal processes, which determine the magnetic configuration of the FM layers, is still lacking. This is due to experimental limitations. Reported experiments rely on magnetization or MR hysteresis curves acquired independently ${ }^{1-11}$ or in measurements performed at a fixed angle of the applied field, normally close to the easy axis (e.a.) direction, recording only the parallel component of the magnetization curve. As a consequence, widely different field-dependent magnetoresistive behaviors, including maximum MR values and curve shapes, are unexpectedly found for multilayers with similar structures.

To tackle these limitations, we employ a magnetoresistanceoptical Kerr effect [M(R)-OKE] setup that allows us to determine simultaneously magnetoresistive responses and magnetization reversal processes. In this paper, we show that both amplitudes and shapes of the MR curves of a spin-valve structure, i.e., a magnetically free-FM layer, a NM spacing layer, and a pinned-FM layer, which is exchange-coupled to an antiferromagnetic (AFM) layer, ${ }^{4}$ depend on the orientation of the applied magnetic field and are directly related to the magnetization reversal processes. The results directly show that the different MR behaviors originate from the magnetic anisotropy of the structure, which ultimately depends on the relative magnetization orientation of the FM layers.

The experimental $\mathrm{M}(\mathrm{R})-\mathrm{OKE}$ setup and the spinvalve structure used in this study are schematically shown in Fig. 1(a). The layer sequence $\mathrm{Ni}_{80} \mathrm{Fe}_{20}(9 \mathrm{~nm}) /$ $\mathrm{Cu}(2 \mathrm{~nm}) / \mathrm{Ni}_{80} \mathrm{Fe}_{20}(9 \mathrm{~nm}) / \mathrm{FeMn}(15 \mathrm{~nm})$ was grown at room temperature (RT) by sputtering on an oxidized $\mathrm{Si}$ substrate precovered with a 2-nm-thick Ta buffer layer. ${ }^{12}$ The free$\mathrm{FM} / \mathrm{Cu} /$ pinned-FM/AFM structure was then capped with a 2-nm-thick Ta layer to prevent oxidation. The sample was heated up to $420 \mathrm{~K}$ for $30 \mathrm{~min}$ and then field-cooled (FC) to $\mathrm{RT}$ in a magnetic field of $250 \mathrm{mT}$. This procedure locks the magnetization of the pinned-FM layer along the FC direction via the induced unidirectional anisotropy arising from the interfacial exchange coupling with the adjacent AFM FeMn layer, and it defines the magnetization e.a. direction of the system.

Magnetization reversal processes and magnetoresistive properties were studied at RT by measuring simultaneously in-plane vectorial-resolved magnetization hysteresis loops and the corresponding resistance changes. We have employed a four-probe ac technique with the applied current flowing parallel to the e.a. of the system. The sample was contacted using Al-wire bonding in a linear four-probe geometry and the measurements were performed in current-in-plane (CIP) geometry using a lock-in amplifier. An ac current of about $50 \mu \mathrm{A}$, with a frequency of $75 \mathrm{kHz}$, was provided by a wave form generator through a load resistance of $R_{\mathrm{L}} \approx$ $6 \mathrm{k} \Omega$, whereas the sample resistance is about $10 \Omega$. The magnetoresistance curves $\operatorname{MR}(H)=\frac{R(H)-R_{0}}{R_{0}}$ (where $R_{0}$ is the resistance in the $\mathrm{P}$ state) were hence extracted from the experimental $R(H)$. The magnetization loops were measured by high-resolution vectorial-Kerr magnetometry by using $p$-polarized light focused between the inner electric probes and 
(a)

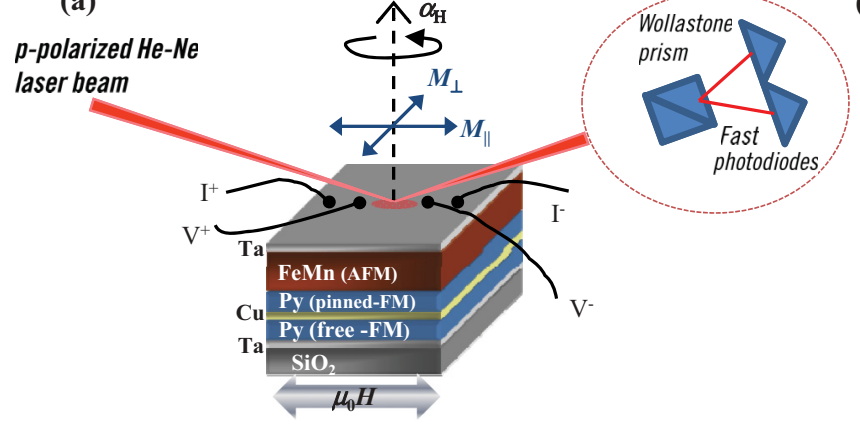

(b)

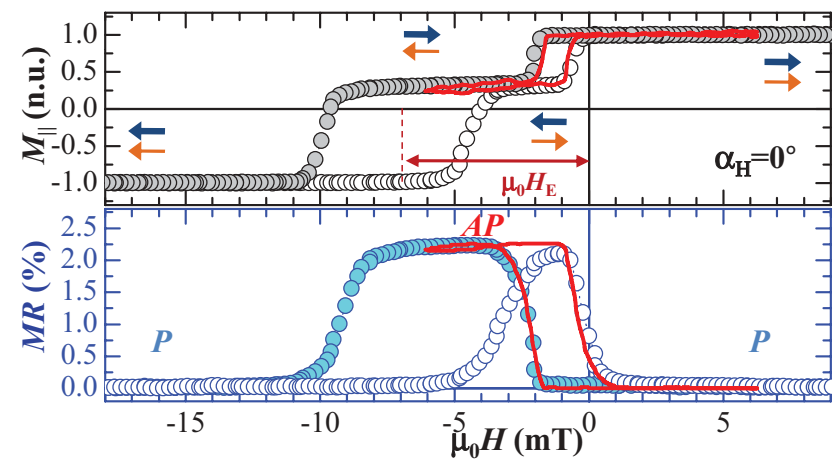

FIG. 1. (Color online) (a) Scheme of the experimental M(R)-OKE setup combining simultaneous vectorial-Kerr and magnetoresistance capabilities and the spin-valve structure investigated. (b) $M_{\|}-H$ (top graph) and MR- $H$ (bottom) hysteresis curves acquired simultaneously at the e.a. direction, i.e., $\alpha_{\mathrm{H}}=0^{\circ}$. The symbols (lines) correspond to the major, i.e., $\pm 20 \mathrm{mT}$ (minor, $\pm 6 \mathrm{mT}$ ) hysteresis loop. The two branches of the hysteresis are depicted with filled and empty symbols for decreasing and increasing fields, respectively. Parallel (P) and antiparallel (AP) magnetic configurations are indicated. Notice the correspondence between magnetization and magnetoresistive hysteresis loops.

analyzing the two orthogonal components of the reflected light. This provides the (additional) simultaneous determination of the hysteresis loops of both in-plane parallel, $M_{\|}$, and transverse, $M_{\perp}$, magnetization components as a function of the applied magnetic field. ${ }^{13}$ The angular-dependent study has been performed as a function of the sample in-plane angular rotation angle $\alpha_{\mathrm{H}}$, keeping fixed the external magnetic field direction. The whole angular range was probed every $1.8^{\circ}$, with $0.5^{\circ}$ angular resolution.

The capability of the M(R)-OKE setup is shown in Fig. 1(b). Both magnetization $M_{\|}(H)$ (for simplicity the transverse component is not shown here) and magnetoresistance $\operatorname{MR}(H)$ major loops $( \pm 20 \mathrm{mT})$ acquired simultaneously at the e.a. direction, i.e., $\alpha_{\mathrm{H}}=0^{\circ}$, present two distinctive hysteresis loops, which can be assigned to the intrinsic magnetization reversal behavior of the two FM layers. The free-FM layer exhibits an individual hysteresis loop with a residual shift from the zero field ( $\sim-1.2 \mathrm{mT})$, which might originate from a magnetostatic "orange-peel" coupling with the pinned-FM layer, ${ }^{14}$ and low coercivity $(\sim 0.9 \mathrm{mT})$. The pinned-FM layer presents another individual hysteresis loop shifted away from the zero magnetic field $\left(\mu_{0} H_{\mathrm{E}} \approx-7 \mathrm{mT}\right)$ due to the unidirectional exchange anisotropy with the adjacent AFM layer ${ }^{15}$ and larger coercivity (3 mT).

To correlate the resistance changes with the magnetization reversal of both FM electrodes, we compare the MR curve (bottom graph) with the magnetization loop (top graph). First, sharp transitions are observed at similar magnetic field values in both curves, as Fig. 1(b) shows. Second, the MR curve changes from low to high, and finally low, resistance states when the magnetic field is swept, independently from the field branch. Well-defined plateaus with maximum value of about $2.3 \%$ are found in both descending and ascending field branches, but much wider in the former. These features can be explained with the magnetization configuration of the FM layers, as labeled in Fig. 1(b). The magnetic field range where the magnetization orientation of the two FM layers is AP is much smaller for the ascending branch. Similarly, the magnetization changes of the minor loop $( \pm 6 \mathrm{mT}$, depicted with lines) are associated mainly with a magnetic reorientation of the free-FM layer, i.e., the pinned-FM layer does not reverse, and the MR just changes from its minimum to its maximum value for the descending branch, and vice versa for the ascending one.

Figure 2 shows representative in-plane resolved magnetization ( $M_{\|}$and $M_{\perp}$ components) and MR hysteresis curves acquired at selected $\alpha_{\mathrm{H}}$ angles around the characteristic e.a. and hard axis (h.a.) directions. The complete angular-dependent study is presented in the Supplemental information. ${ }^{16}$ At first glance, different magnetization reversal and magnetoresistive responses are clearly distinguished when the angle of the applied field is changed from the e.a. to the h.a. direction.
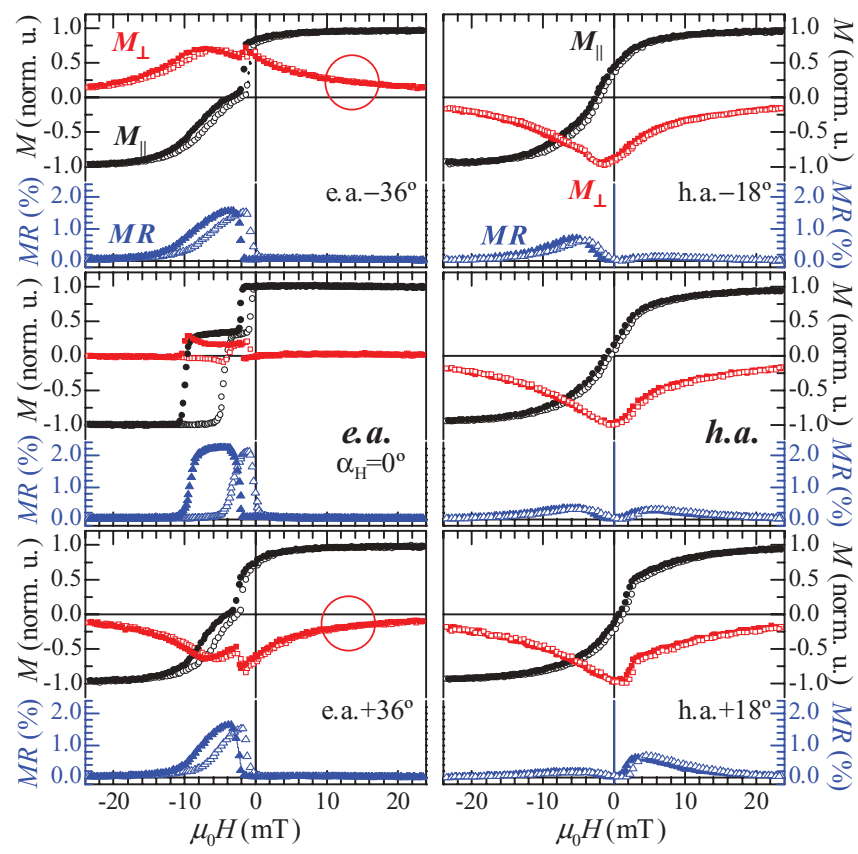

FIG. 2. (Color online) Selected in-plane resolved magnetization and MR curves of the spin-valve structure around the e.a. (left graphs) and h.a. (right) directions. The corresponding angles of the applied field $\alpha_{\mathrm{H}}$ are indicated in the graphs. The experimental $M_{\|}\left(H, \alpha_{\mathrm{H}}\right)$, $M_{\perp}\left(H, \alpha_{\mathrm{H}}\right)$, and $\operatorname{MR}\left(H, \alpha_{\mathrm{H}}\right)$ loops are represented by circles, squares, and triangles, respectively. The ascending (descending) branches are displayed with filled (open) symbols. 
In general, similar symmetries and magnetic field transitions are observed in both magnetization and MR curves, which indicates their direct relationship.

A simple inspection of the $M_{\|}$and $M_{\perp}$ hysteresis curves provides information about the magnetization reversal processes and the magnetic anisotropy, highlighting the importance of vectorial magnetometry measurements. The hysteresis curves present sharp (irreversible) and round (reversible) transitions, and their relative weight depends on $\alpha_{\mathrm{H}}$. The former are more relevant close to the e.a. direction, whereas reversible transitions become increasingly important away from it. Close to the h.a. direction, only reversible transitions are found. As expected for extended magnetic systems, the irreversible transitions correspond to nucleation and further propagation of magnetic domains, ${ }^{13}$ whereas the reversible transitions correspond to rotation processes. Note that the rotation processes are easier to identify in the $M_{\perp}$ hysteresis loops, which also provide relevant information on the magnetization configuration between the FM layers and on the magnetic anisotropy. The nonvanishing $M_{\perp}$ curve found at the e.a. direction (central left graph of Fig. 2) indicates that the relative magnetic orientation of the two FM layers is not fully AP. The $M_{\perp}$ hysteresis loops change sign around the e.a. direction (top and bottom left graphs), but their sign is preserved across the h.a. direction. The same behavior (with opposite sign) is found by rotating the sample by $180^{\circ}$ (see Ref. 16). This indicates that the reversal is dictated by the induced unidirectional anisotropy of the pinned-FM layer. ${ }^{17}$

The magnetoresistive response is correlated with the magnetization curves. For instance, the maximum value of MR and the widest MR plateau (in field) are found at the e.a. direction $\left(\alpha_{\mathrm{H}}=0^{\circ}\right)$, i.e., where the magnetization reversal is mainly governed by irreversible processes and a better AP alignment between the two FM layers is achieved. Although well-defined MR plateaus and relatively high MR values are still found away from the e.a. direction, they decrease progressively as the field is misaligned with respect to the e.a. direction, i.e., when reversible magnetization processes become important. Upon approaching the h.a. direction, only reversible transitions are found in both magnetization and MR. The lowest resistivity changes are observed at the h.a. direction, and are one order of magnitude smaller than those at the e.a. direction.

The correlation, however, is not complete. While all sharp magnetization transitions are associated with similar transitions in the MR curves, not all round magnetization transitions result in relevant MR changes. For instance, magnetization loops acquired at $\pm 36^{\circ}$ (top and bottom left graphs of Fig. 2) presents similar pronounced round magnetization transitions for both positive and negative magnetic fields values (clearly observed in the transverse component), but for positive field the round magnetization transitions do not correspond to significant MR changes (circles in top and bottom left graphs). A simultaneous magnetization rotation of both FM layers during positive fields could explain this observation. In this case, an anisotropic magnetoresistance (AMR) effect would be expected from the whole spin-valve structure, but it is much smaller than the GMR effect originating from the relative magnetization reorientation between the FM layers.

To identify the spin-dependent transport process at the origin of the magnetoresistive signal, we have exploited our
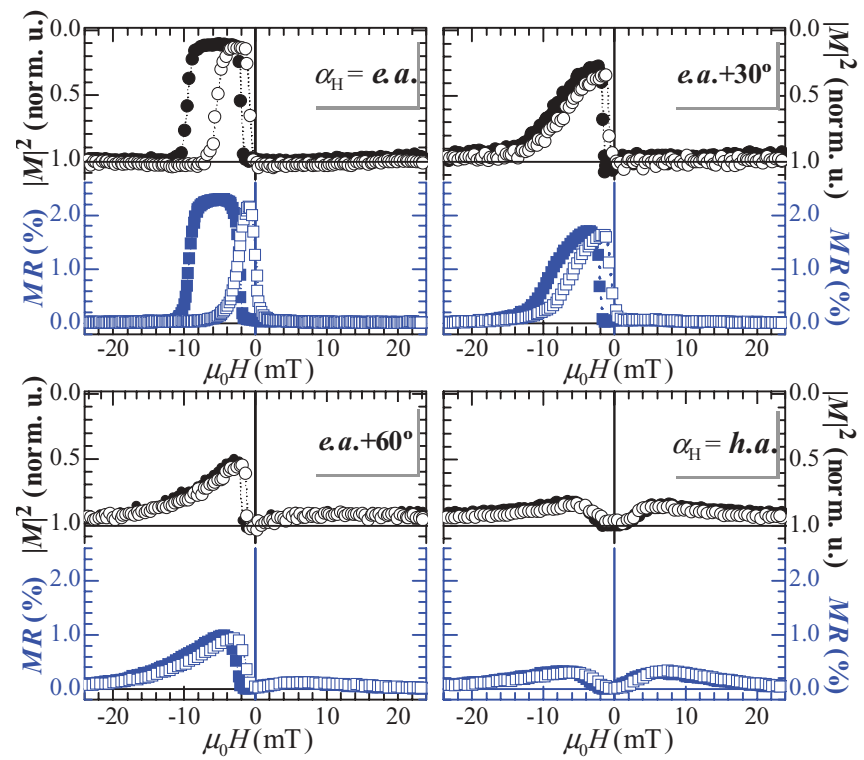

FIG. 3. (Color online) Comparison between the square modulus of magnetization (top graphs) and the magnetoresistance (bottom graphs) hysteresis curves at selected $\alpha_{\mathrm{H}}$, from the e.a. to the h.a. direction (from upper left to bottom right). Notice that $\operatorname{MR}(H)$ and $|M(H)|^{2}$ behave similarly for all angles.

vectorial magnetometry data by studying the dependence of the MR curves with the angle $\phi$ between the magnetization of the FM layers, independently of the angular orientation of the external magnetic field. We recall that the square modulus of the magnetization, as derived from the in-plane resolved magnetization loops of the parallel and transverse components, is proportional to $\cos \phi$, i.e., $|M|^{2}=M_{\|}^{2}+M_{\perp}^{2} \propto \cos \phi$. The simultaneous determination of both components is, obviously, crucial to obtain the square modulus. Figure 3 compares $|M(H)|^{2}$ (top) and $\operatorname{MR}(H)$ (bottom) curves at representative angles of the applied field. The comparison for all angles can be found in the Supplemental information. ${ }^{16}$ Noticeably, $|M(H)|^{2}$ and $\mathrm{MR}(H)$ present strikingly similar features (hysteresis shapes, maximum values, etc.) for all angles. For instance, at the e.a. direction the MR jumps coincide exactly with the abrupt changes of the magnetization modulus, while sharp and round transitions (with lower maxima) appear simultaneously away from the e.a. direction. In addition, similar symmetries are found in both hysteresis curves. This can only be explained by assuming that the magnetoresistive response depends strictly on the relative magnetization orientation between the FM electrodes for the whole angular range.

Several experimental works have dealt with the dependence of the MR with $\phi \cdot{ }^{4,8,9,11}$ In contrast to the present data, however, $\phi$ was derived from a single hysteresis curve acquired at the expected e.a. direction and by assuming that the pinned-FM layer is fixed and only the free-FM layer reorients its magnetization. These assumptions are not necessarily true. MR measurements performed in CIP geometry showed a linear relationship with $\cos \phi$, as expected from spin-dependent scattering processes in the bulk of the FM layers, whereas significant deviations were reported for measurements in the current-perpendicular-to-plane (CPP) geometry. ${ }^{18}$ The nonlinearities were associated with asymmetrical features of 
the structure, such as different FM layers, spin-dependent potentials at interfaces, and device boundaries. ${ }^{10,19}$ Our CIP geometry results show directly that the linear behavior of MR with $\cos \phi$ is valid in the whole angular range. This is direct experimental evidence that the magnetoresistive response depends on the intrinsic anisotropy of a multilayer structure through its angular-dependent magnetization reversal processes, and, ultimately, that it relies on the relative orientation of the FM layers. In addition, our simultaneous magnetization reversal study shows that the assumption of the fixed magnetization orientation of the pinned-FM layer is only valid at the e.a. direction, revealing the importance of direct experimental access to the angle $\phi$, and suggesting the need to reexamine the real influence of possible additional (nonlinear) contributions to the MR behavior by measuring the angle $\phi$ properly.

In summary, we have shown that the magnetoresistive response of a spin-valve structure when exposed to an external field varying in magnitude and/or direction presents different fingerprints that depend sensitively on the details of the magnetization reversal processes dictated by the intrinsic magnetic anisotropy of the system. To this end, we have determined simultaneously the magnetization reversal processes (with vectorial resolution) and the MR hysteresis loops of magnetic nanostructures as a function of the applied field direction for the whole angular range. Remarkably, the hysteresis loops of the square modulus of the magnetization, which reflects the relative magnetic orientation between the free-FM and the pinned-FM layers, reproduce the shape and details of the MR curves for all angles, confirming the models based on bulk spin-dependent scattering processes. Our results show unambiguously that the widely different field-dependent magnetoresistive behaviors originate from extrinsic parameters, such as magnetic field orientations, and/or intrinsic magnetic anisotropies.

This work was supported in part by the Spanish MICINN through Project No. CSD2007-00010 and FIS 2010-18847 and by the Comunidad de Madrid through Project No. S2009/MAT1726. P.P. acknowledges support through the Marie Curie AMAROUT EU action and the Spanish MICINN "Juan de la Cierva" contract. A.B. acknowledges support through the "Ramón y Cajal" contract from the Spanish MICINN. *paolo.perna@imdea.org

†julio.camarero@uam.es

${ }^{1}$ M. N. Baibich, J. M. Broto, A. Fert, F. Nguyen Van Dau, F. Petroff,

P. Etienne, G. Creuzet, A. Friederich, and J. Chazelas, Phys. Rev. Lett. 61, 2472 (1988); G. Binasch, P. Grünberg, F. Saurenbach, and W. Zinn, Phys. Rev. B 39, 4828 (1989).

${ }^{2}$ G. A. Prinz, Science 282, 1660 (1998).

${ }^{3}$ A. Fert, Angew. Chem. Int. Ed. 47, 5956 (2008); C. Chappert,

A. Fert, and F. N. Van Dau, Nat. Mater. 6, 813 (2007).

${ }^{4}$ B. Dieny, V. S. Speriosu, S. S. P. Parkin, B. A. Gurney, D. R. Wilhoit, and D. Mauri, Phys. Rev. B 43, 1297 (1991).

${ }^{5}$ J. S. Moodera, L. R. Kinder, T. M. Wong, and R. Meservey, Phys. Rev. Lett. 74, 3273 (1995)

${ }^{6}$ J. A. Katine, F. J. Albert, R. A. Buhrman, E. B. Myers, and D. C. Ralph, Phys. Rev. Lett. 84, 3149 (2000).

${ }^{7}$ J. Grollier, V. Cros, A. Hamzic, J. M. George, H. Jaffrès, A. Fert, G. Faini, J. Ben Youssef, and H. Legall, Appl. Phys. Lett. 78, 3663 (2001).

${ }^{8}$ L. B. Steren, A. Barthelemy, J. L. Duvail, A. Fert, R. Morel, F. Petroff, P. Holody, R. Loloee, and P. A. Schroeder, Phys. Rev. B 51, 292 (1995).

${ }^{9}$ A. Chaiken, G. A. Prinz, and J. J. Krebs, J. Appl. Phys. 67, 4892 (1990).

${ }^{10}$ J. Barnás, O. Baksalary, and A. Fert, Phys. Rev. B 56, 6079 (1997).

${ }^{11}$ D. Y. Kim, C. G. Kim, B. S. Park, D. G. Hwang, and S. S. Lee, IEEE Trans. Magn. 35, 2934 (1999).

${ }^{12}$ M. Romera, M. Muñoz, P. Sánchez, C. Aroca, and J. L. Prieto, J. Appl. Phys. 106, 023922 (2009).
${ }^{13}$ J. Camarero, J. Sort, A. Hoffmann, J. M. García-Martín, B. Dieny, R. Miranda, and J. Nogués, Phys. Rev. Lett. 95, 057204 (2005).

${ }^{14}$ Y. Pennec, J. Camarero, J.-C. Toussaint, S. Pizzini, M. Bonfim, F. Petroff, W. Kuch, F. Offi, K. Fukumoto, F. Nguyen Van Dau, and J. Vogel, Phys. Rev. B 69, 180402 (2004).

${ }^{15}$ D. Ecija, E. Jiménez, N. Mikuszeit, N. Sacristán, J. Camarero, J. M. Gallego, J. Vogel, and R. Miranda, Phys. Rev. B 77, 024426 (2008); J. Magn. Magn. Mater. 316, 321 (2007).

${ }^{16}$ See Supplemental Material at http://link.aps.org/supplemental/ 10.1103/PhysRevB.86.024421 for an animation showing the complete angular dependent $\mathrm{M}(\mathrm{R})-\mathrm{OKE}$ study. The animation illustrates $\mathrm{M}(\mathrm{R})$-OKE measurements at different applied magnetic field angles $\alpha_{\mathrm{H}}$ with respect to the e.a. direction, i.e., as rotating the sample. For a given angle, vectorial-resolved magnetization (a) and magnetoresistance (b) hysteresis curves acquired simultaneously, as well as the corresponding square modulus of magnetization (c) hysteresis curves, are shown. It is worth noting the direct connection between magnetization reversal and magnetoresistive response as well as that $\mathrm{MR}(H)$ and $|M(H)|^{2}$ behave similarly in the whole angular range.

${ }^{17}$ E. Jiménez, J. Camarero, J. Sort, J. Nogués, A. Hoffmann, F. J. Teran, P. Perna, J. M. García-Martín, B. Dieny, and R. Miranda, Appl. Phys. Lett. 95, 122508 (2009).

${ }^{18}$ P. Dauguet, P. Gandit, J. Chaussy, S. F. Lee, A. Fert, and P. Holody, Phys. Rev. B 54, 1083 (1996).

${ }^{19}$ A. Vedyanev, B. Dieny, N. Ryzhanova, J. B. Genin, and C. Cowache, Europhys. Lett. 25, 465 (1994). 